\title{
Assessing Machine Learning versus Mathematical Modeling to Estimate the Transverse Shear Stress Distribution in a Rectan- gular Channel
}

\author{
Babak Lashkar-Ara ${ }^{1, *}$, Niloofar Kalantari ${ }^{1}$, Zohreh Sheikh Khozani ${ }^{2}$ and Amir Mosavi ${ }^{2,3,4, *}$ \\ 1 Department of Civil Engineering, Jundi-Shapur University of Technology, Dezful, Iran \\ 2 Institute of Structural Mechanics, Bauhaus Universität-Weimar, 99423 Weimar, Germany \\ 3 School of Economics and Business, Norwegian University of Life Sciences, 1430 Ås, Norway \\ 4 John von Neumann Faculty of Informatics, Obuda University, 1034 Budapest, Hungary \\ * Correspondence: Lashkarara@jsu.ac.ir; amir.mosavi@mailbox.tu-dresden.de
}

\begin{abstract}
One of the most important subjects of hydraulic engineering is the reliable estimation of the transverse distribution in rectangular channel of bed and wall shear stresses. This study makes use of the Tsallis entropy, Genetic Programming (GP) and adaptive neuro-fuzzy inference system (ANFIS) methods to assess the shear stress distribution (SSD) in rectangular channel. To evaluate the results of the Tsallis entropy, GP and ANFIS models, laboratory observations were used in which shear stress was measured using an optimized Preston tube. This is then used to measure the SSD in various aspect ratios in the rectangular channel. To investigate the shear stress percentage, 10 data series with a total of 112 different data for were used. The results of the sensitivity analysis show that the most influential parameter for the SSD in smooth rectangular channel is the dimensionless parameter $B / H$, Where the transverse co-ordinate is $B$, and the flow depth is $H$. With the parameters $(b / B),(B / H)$ for the bed and $(z / H),(B / H)$ for the wall as inputs, the modeling of the GP was better than the other one. Based on the analysis, it can be concluded that the use of GP and ANFIS algorithms is more effective in estimating shear stress in smooth rectangular channels than the Tsallis entropy-based equations.
\end{abstract}

Keywords: Smooth rectangular channel; Tsallis entropy; genetic programming; artificial intelligence; machine learning; big data; computational hydraulics; neuro fuzzy

\section{Introduction}

Knowledge of boundary shear stress is necessary when studying sediment transport, flow pattern around structures, estimation of scour depth and channel migration. The determination of boundary shear stress, i.e. at the wall and bed depends on the channel geometry and its associated roughness. Various direct and indirect methods have been extensively discussed in experimentally measure the wall and bed shear stresses in channels with different cross sections [1-4]. Bed shear stress can be estimated based on four techniques (1) bed slope product $\tau_{b}=g H S$, (2) law of the wall velocity profiles $\frac{u}{u_{*}}=\frac{1}{k \times} \ln \left(\frac{z}{z_{0}}\right)$ where $\tau_{b}=\rho u_{*}^{2}$, (3) Reynolds stress measurement $\tau_{b}=\rho\left(-u^{\prime} w^{\prime}\right)$, (4) Turbulent Kinetic Energy (TKE), TKE $=\frac{\rho\left(\overline{u^{\prime 2}}+\overline{v^{\prime} 2}+\overline{w^{\prime 2}}\right)}{2}$, where $\tau_{b}=C_{1} T K E$, where $u^{\prime}, v^{\prime}$ and $w^{\prime}$ are the fluctuating horizontal, transversal and vertical velocity components, respectively and $C_{1}=0.20$ [5]. The symbols $g, \mathrm{H}$ and $S$ denote gravity, water level and channel slope, respectively, whereas $u$ is the velocity at height $\mathrm{z}, u_{*}$ is the shear velocity, $k$ is von Karman constant, and $z_{0}$ is the roughness length. 
These methods are useful in presenting a point-based representation of shear stress in a channel, whereas the shear stress distribution (SSD) provides a more accurate hydrodynamic profile within a channel. Knight and Sterling [6] measured the SSD in a circular channel with and without sediment. They examined a wide range of flow depths for each level benching and therefore it had been possible to determine the extent to which the hydraulics changes Park et al. [7] utilized laboratory-scale water flume and measured the bed shear stress under high-velocity flow conditions directly.

Lashkar-Ara and Fatahi [8] measured transverse SSD in the channel bed and wall by using an optimal diameter Preston tube to evaluate the SSD on a rectangular open channel. The outcome of this research is two-dimensional relationships to evaluate local shear stress in both bed and wall. The bed and wall relative coordinates $b / B$ and $z / H$ in the cross section also the aspect ratio $B / H$ are the function of these relationships. The study showed that the dimensionless SSD is greatly affected by the aspect ratio.

Utilizing the advantages offered in the soft computing method and the Artificial Intelligence (AI) techniques, other researchers have been extended numerically and analytically to overcome difficulties with experimental measurements [9-12]. Martinez-Vazquez and Sharifi [13] utilized recurrence plot (RP) analysis and Eigenface for Recognition to estimate the SSD in trapezoidal and circular channels. A new approach has been developed by Sterling and Knight [14] to estimate the SSD in a circular open channel. In terms of accuracy, the analysis showed that there is a lack of ability in the outcome and it is not satisfactory. The uncertainty of the estimation of the model parameters and the high sensitivity of the outcomes to the expected experiment parameters can be due to this.

Sheikh Khozani and Bonakdari [15] extended the analytical method based Renyi entropy to estimate SSD in circular channels. Sheikh Khozani and Bonakdari [16] researched on the comparison of five different models in straight compound channel prediction of SSD. In other research, Sheikh Khozani and Wan Mohtar [10] analyzed the formulation of the SSD on the basis of the Tsallis entropy in circular and trapezoidal channels. Sheikh Khozani et al. [17] have attempted in another study to use an improved SVM method to estimate shear stress in rough rectangular channel.

Ardiçlioğlu et al.[18], conducted an experimental study for the SSD throughout the entire length of the cross-section in fully developed boundary layer area, in an open rectangular channel, in both smooth and rough surface. By measuring the speed in both smooth and rough surfaces, they conducted tests. Using logarithmic distribution of velocity, the average shear stresses in the cross section for aspect ratios of 4.2 to 21.6 and the Froude numbers of 0.12 to 1.23 were measured. The definition of the Tsallis entropy was used by Bonakdari et al. [19] to predict the SSD in trapezoidal and circular channels and achieve acceptable accuracy. Although the direct measurement of shear stress in laboratory provides correct description of the spatial pattern, the measurement of shear stress using shear place or cell is laborious, complex, requires careful calibration and may not applicable to all type of channels [20]. The use of soft computing techniques in the simulation of engineering problems was intensively studied and a variety of soft computing methods were suggested. To approximate the daily suspended sediment load, Kisi et al. [21] used a GP model. They also contrasted this approach with several soft computing approaches and concluded that the GP model works better than the others. In estimating SSD in circular channels with and without flat-bed Sheikh Khozani et al. [22,23] applied Randomize Neural Network (RNN) and Gene expression Programming (GEP).

In this study, the Tsallis entropy was used to determine SSD in a smooth bed and wall in a rectangular open channel. This is then used to measure the SSD in various aspect ratios in the rectangular channel. In the second part of the study, two soft computing methods were applied to predict the transverse of SSD in the smooth rectangular channel. The methods of genetic programming (GP) and (ANFIS) were examined to determine the precision of these models in estimating bed and wall shear stress. This study aimed at using Tsallis entropy method to predict the SSD in smooth rectangular channel. The results of the Tsallis entropy, GP and ANFIS methods compared with experimental results of Lashkar-Ara and Fatahi [8]. Although this analysis was performed in parallel with 
Sheikh Khozani and Bonakdari [16] research, it can be said in a practical contrast that the data used in this study is based on the measurement of shear stress using the optimal diameter of the Preston tube, which was designed by Lashkar-Ara and Fatahi [8], so the comparison of findings is more precise and less uncertain.

\section{Materials and Methods}

\subsection{Data collection}

Information on the SSD has been collected in the Lashkar-Ara and Fatahi [8] experiments of a smooth rectangular channel, performed in a flume 10-meter long, $60 \mathrm{~cm}$ wide and $70 \mathrm{~cm}$ high. All measurements were performed in the range of 11.06 to 102.38 liter per second flow rate. Flow rate variations led to observable changes in water depth ranging from $4.3 \mathrm{~cm}$ to $21 \mathrm{~cm}$ and the aspect ratio of 2.86 to 13.95 . The values of static and total pressure difference in various aspect ratios of $B / H$ were measured and reported using pressure transducer apparatus with a capacity of 200 mill bar and $50 \mathrm{~Hz}$ measuring frequency. In order to create uniform flow condition and to match the hydraulic gradient with the flume bed slope a weir at the end of the flume was installed. Figure 1 illustrates the notation used for a smooth rectangular channel conduit. Figure 2 shows the schematic of experimental setup.

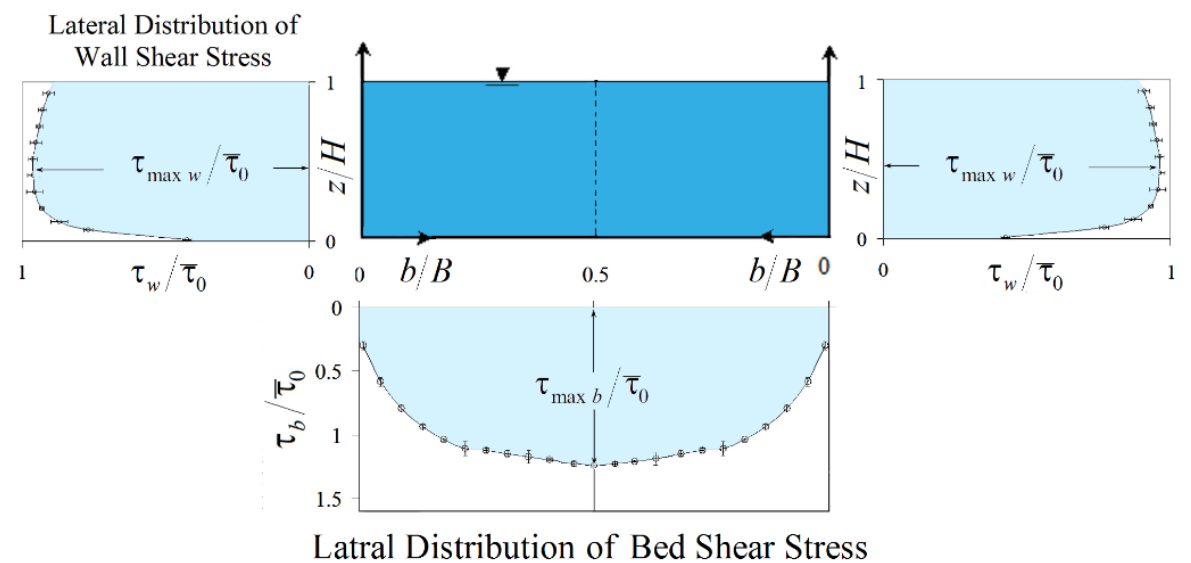

Figure 1. Schematics of local SSD coordinates in the rectangular channel wall and bed.

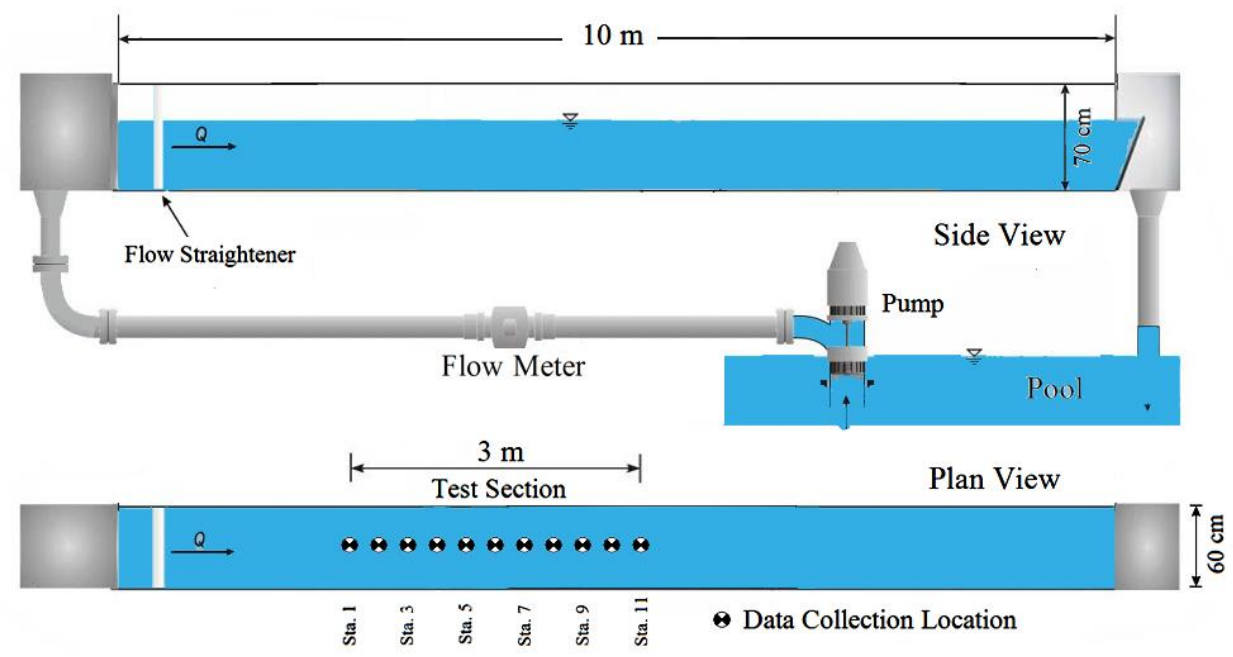

Figure 2. Experiment schematic.

Based on previous studies in laboratory and field investigation, the effective criteria for evaluating the SSD along the wet periphery of a channel can be expressed as follows: 


$$
\begin{aligned}
& f_{1}\left(\bar{\tau}_{w}, \rho, v, g, V, H, S_{w}, S_{o}, B, K_{S}\right)=0 \\
& f_{1}\left(\bar{\tau}_{b}, \rho, v, g, V, H, S_{w}, S_{o}, B, K_{S}\right)=0
\end{aligned}
$$

where $\bar{\tau}_{w}$ is the average wall shear stress, $\bar{\tau}_{b}$ is the average bed shear stress, $\rho$ is the density, $v$ is the kinematic viscosity, $g$ is the gravity acceleration, $V$ is the flow velocity, $H$ is the flow depth, $B$ is the Flume floor width, $S_{w}$ is the Water surface slope, $k_{s}$ is the roughness height, $(\mathrm{Re})$ is the Reynolds number and (Fr) is the Froude number.

The Buckingham- $\pi$ theorem is used to obtain independent dimensional parameters for wall and bed shear stress, as shown in Eq. (3) and Eq. (4).

$$
\begin{aligned}
& f\left(\frac{v}{V H}, \frac{K_{s}}{H}, \frac{g H}{V^{2}}, \frac{B}{H}, \frac{\bar{\tau}_{w}}{\rho g H S_{w}}\right)=0 \\
& f\left(\frac{v}{V H}, \frac{K_{s}}{H}, \frac{g H}{V^{2}}, \frac{B}{H}, \frac{\bar{\tau}_{b}}{\rho g H S_{w}}\right)=0
\end{aligned}
$$

Equations (3) and (4) can be rewritten as (5) and (6):

$$
\begin{aligned}
& \frac{\bar{\tau}_{w}}{\rho g H S_{w}}=f_{3}\left(\operatorname{Re}, F r^{2}, \frac{K_{s}}{H}, \frac{B}{H}, \frac{z}{H}\right) \\
& \frac{\bar{\tau}_{b}}{\rho g y S_{w}}=f_{4}\left(\operatorname{Re}, F r^{2}, \frac{K_{s}}{H}, \frac{B}{H}, \frac{b}{B}\right)
\end{aligned}
$$

For GP simulation, 160 data of bed shear stress $(\tau b)$ and 100 data of wall shear stress $(\tau w)$ were collected in a smooth rectangular channel with different flow depths. Approximately 70 percent of the total data were chosen for training and the remaining 30 percent for testing. The summary of experiments is tabulated in Table 1.

Table 1. Experimental summary

\begin{tabular}{c|cccc}
\hline parameters & Variable definition & minimum & maximum & Mean \\
\hline$H(\mathrm{~m})$ & Flow depth & 0.043 & 0.21 & 0.0928 \\
$B / H$ & aspect ration & 2.86 & 13.95 & 7.98 \\
$Q(\mathrm{~L} / \mathrm{s})$ & Discharge & 11.06 & 102.38 & 34.795 \\
$V(\mathrm{~m} / \mathrm{s})$ & Velocity & 0.429 & 0.813 & 0.568 \\
$\mathrm{Fr}$ & Froude number & 0.66 & 0.566 & 0.618 \\
$\operatorname{Re} \times 10^{4}$ & Reynolds number & 6.4 & 39.87 & 16.418 \\
$\mathrm{Re}^{*}$ & Shear Reynolds & 0.322 & 0.609 & 0.426 \\
$\gamma H S$ & Total shear stress & 0.442 & 2.162 & 0.955 \\
\hline
\end{tabular}

\subsection{Tsallis entropy}

If a random variable $(\tau)$ in a cross section of a channel is assumed to be a consistent shear stress, then, according to Tsallis entropy of [24] the SSD or shear stress probability density function $\mathrm{f}(\tau)$, can be identified as [19]:

$$
H(\tau)=\frac{1}{q-1} \int_{0}^{\tau_{\max } \int} f(\tau)\left(1-f(\tau)^{q-1}\right.
$$

where $\tau$ is the shear stress, $q$ is a true number, and Tsallis 's entropy function is $H(\tau)$. The $\tau$ value varies from 0 to $\tau_{\max }$, and with these restrictions, the integral value of $H(\tau)$ will be 1 .

Using the maximum entropy theorem, the $f(\tau)$ can be calculated to maximize the entropy function subject to specified constraints like Eqs. (8) and (9) respectively [25]. 


$$
\begin{aligned}
& \int_{0}^{\tau_{\max } \int} f(\tau) d \tau=1 \\
& \int_{0}^{\tau_{\max } \int} \tau . f(\tau) d \tau=\bar{\tau}
\end{aligned}
$$

where the mean and maximum shear stress values are $\bar{\tau}$ and $\tau_{\max }$, respectively.

At this stage, using maximization of Lagrange coefficients by Eqs. (7) to (9), the Lagrange function $\mathrm{L}$ can be written down as Eq. (10):

$$
L=\int_{0}^{\left.\tau_{\max }{ }^{q-1}{ }_{0}\left(\int_{0}^{\tau_{\max } S} f(\tau) d \tau-1\right)_{1}\left(\int_{0}^{\tau_{\max } S} \tau \cdot f(\tau) d \tau-\bar{\tau} O\right)\right)} \frac{f(\tau)}{q-1}
$$

where $\lambda_{0}$ and $\lambda_{1}$ are the Lagrange multipliers. By $\partial \mathrm{L} / \partial(\tau)=0$ to maximize entropy, the $f(\tau)$ yields as:

$$
f(\tau)=\left[\frac{q-1}{q}\left(\lambda^{\prime}+\lambda_{1} \cdot \tau\right)\right]^{\frac{1}{(q-1)}}
$$

Which $\lambda^{\prime}=\frac{1}{q-1}+\lambda_{0}$. In Eq. (10), the shear stress probability distribution function (PDF) is represented by $f(\tau)$. The SSD's Cumulative Distribution Function (CDF) is introduced as Eq. (12):

$$
F(\tau)=\int_{0}^{\tau_{\max } \int} f(\tau) d \tau=\frac{y}{L}
$$

where $y$ is the direction of the channel wall, which varies from 0 at the free surface to $L$, and $L$ is the entire wetted perimeter. The function of $f(\tau)$ is the derivative of $F(\tau)$, so a partial derivation of $F(\tau)$ with respect to $y$ is carried out in the following equation:

$$
f(\tau)=\frac{d F(\tau)}{d u}=\frac{1}{L} \frac{d y}{d \tau}
$$

By substituting Eq. (11) into Eq. (12) and Eq. (13) and solving the integral and simplifying, the shear stress function is represented as Eq. (14).

$$
\tau=\frac{k}{\lambda_{1}}\left[\left(\frac{\lambda^{\prime}}{k}\right)^{k}+\frac{\lambda_{1} y}{L}\right]^{\frac{1}{k}}-\frac{\lambda^{\prime}}{\lambda_{1}}
$$

where $k=q / q-1$ and $\mathrm{q}$ is a real parameter in the Tsallis relationship and has an actual value of $3 / 4[10,26] . \lambda_{1}$ and $\lambda^{\prime}$ are Lagrange multipliers that can be derived by trial and error from two implicit equations that follow. Indeed, by inserting and integrating Eq (10) into two constraints (Eqs. (8) and (9)), two Eqs. (15) and (16) are returned as:

$$
\tau 1\left[\lambda^{\prime}+\lambda_{1} \tau_{\max }[]^{k}\left[\lambda^{\prime}+\lambda_{1} \tau_{\max }[]^{k+1}\left[\lambda^{\prime}\right]^{k+1^{2}{ }^{k}}\right]\right]_{\max }
$$

Eqs. (15) and (16) solve to obtain two undefined Lagrange multipliers $\left(\lambda_{1}\right.$ and $\left.\lambda^{\prime}\right)$. To estimate the SSD, a pair of mean and maximum shear stresses is required. The results of the Lashkar-Ara and Fatahi [9] studies have been used for this reason in order to estimate the values of $\tau_{\max }$ and $\bar{\tau}$. They adjusted the slope of the bed flume at $9.58 \times 10^{-4}$. The shear stress carried by the walls and bed was measured for different aspect ratio $(B / H=$ $2.86,4.51,5.31,6.19,7.14,7.89,8.96,10.71,12.24$ and 13.95). For each aspect ratio, the distribution of shear stress in the bed and wall was measured by a Preston tube. Preston's method of measuring boundary shear stress, which makes use of a Pitot tube resting on 
the surface, depends upon the assumption of a region of flow similarity, adjacent to the bed and walls of the channel, common to fully developed turbulent flow and the turbulent boundary layer. Among all the experimental results for $\tau_{\max }$ and $\bar{\tau}$, the best fit equation was obtained separately for bed and wall in aspect ratio $2.89<B / H<13.95$. Relationships are shown in Eqs. (17) to (20).

$$
\begin{gathered}
\frac{\bar{\tau}_{w}}{\rho g R S}=\frac{2.1007+0.0462\left(\frac{B}{H}\right)}{1+0.1418\left(\frac{B}{H}\right)+\left(\frac{B}{H}\right)^{-0.0424}} \\
\frac{\bar{\tau}_{b}}{\rho g R S}=\frac{2.0732-0.0694\left(\frac{B}{H}\right)}{1-0.146\left(\frac{B}{H}\right)+\left(\frac{B}{H}\right)^{-0.1054}} \\
\frac{\tau_{\max \quad w}=\frac{2.5462+6.5434\left(\frac{B}{H}\right)}{1+6.34\left(\frac{B}{H}\right)+\left(\frac{B}{H}\right)^{-0.1083}}}{\frac{3.157+0.8214\left(\frac{B}{H}\right)}{\rho g R S}=\frac{\tau_{\max \quad b}}{1+0.8535\left(\frac{B}{H}\right)+\left(\frac{B}{H}\right)^{-0.1401}}}
\end{gathered}
$$

where $\bar{\tau}_{w} \& \bar{\tau}_{b}$ and $\tau_{\max w} \& \tau_{\max b}$ are the mean and maximum shear stress on the channel wall and bed, respectively. Therefore, the transverse SSD for the rectangular open channel can be determined depending on the aspect ratio and the slope of the channel bed.

\subsection{Genetic Programming (GP)}

In the second part of this analysis, the GP model is applied as one of the evolutionary algorithms (EA) to improve the accuracy of the given relations. The GP is an automated programming method to solve problems by designing computer programs GP is widely used for modeling structure recognition technology applications concerns. For this aim the GP technique is used to understand the basic structure of a natural or experimental process. In the GP method a population is slowly improved by selectively omitting the population who is not so fit and selecting from better species. EAs perform defining a target in the form of a quality criterion and then using that objective to evaluate candidates for a solution in a step-by-step improvement of data set frameworks and returning within a few iterations an optimum solution. The GP can optimize both the structure of the model and its parameters. One of the advantages of the GP algorithm is that it can extract an equation based the input and output parameters and it is more effective than other ANN models [27]. Table 2 represents the used parameters in modeling with GP algorithm.

Table 2. Parameters of the GP models

\begin{tabular}{ccccc}
\hline Parameter & Definition & Value (Model 1) & Value (Model 2) & Value (Model 3) \\
\hline 1 & Function set & $+,-, *, \sqrt{ }, \wedge 2, \cos$, & $+,-, * \sqrt{ }, \wedge 2$, & $+,-, *, \sqrt{ }, \wedge 2$, \\
$2-1$ & The terminal set for $\frac{\tau_{b}}{\bar{\tau}}$ & $b / B, B / H$, Fr, Re & $b / B, B / H$, Fr & $b / B, B / H$ \\
$2-2$ & The terminal set for $\frac{\tau_{w}}{\bar{\tau}}$ & $z / H, B / H$, Fr, Re & $z / H, B / H$, Fr & $z / H, B / H$ \\
3 & Number of inputs & 4 & 3 & 2 \\
4 & The fitness function & RMSE & RMSE & RMSE \\
5 & Error type & error function & error function & error function \\
6 & Crossover rate & $0.55 \%$ & $0.55 \%$ & $0.55 \%$ \\
\hline
\end{tabular}




\begin{tabular}{ccccc}
\hline 7 & Mutation rate & $0.1 \%$ & $0.1 \%$ & $0.1 \%$ \\
8 & Gene reproduction rate & $0.05 \%$ & $0.05 \%$ & $0.05 \%$ \\
9 & Population size & 250 & 250 & 250 \\
10 & Number of generations & 112 & 112 & 112 \\
11 & Tournament type & Regular & Regular & Regular \\
12 & Tournament size & 6 & 6 & 6 \\
13 & Max tree depth & 4 & 4 & 4 \\
14 & Max node per tree & Inf & Inf & Inf \\
15 & Constants range & {$[-10,+10]$} & {$[-10,+10]$} & {$[-10,+10]$} \\
\hline
\end{tabular}

The outcomes of the GP model were analyzed by using the statistical indexes and compared with the experimental results.

\subsection{Adaptive neuro fuzzy inference system (ANFIS)}

ANFIS is designed to provide the requisite inputs and outputs for adaptive networks to build fuzzy rules with acceptable membership functions. ANFIS is a common and cardinal programming method that uses fuzzy theory to write fuzzy if-then rules and fuzzy logic bases that map from a given input information to the desired output. An adaptive network is a multi-layer feed-forward Artificial Neural Network (ANN) with; partially or entirely adaptive nodes in which the outputs are predicted on adaptive node parameters and the parameter adjustment is specified by the learning rules due to the error term. In adaptive ANFIS, hybrid learning is generally a learning form [28].

\subsection{Criteria for Statistical Assessment}

Maximum Error (ME), Mean Absolute Error (MAE), Root Mean Square Error (RMSE) and Nash-Sutcliffe Efficiency (NSE) are the four statistical evaluation parameters used to determine the Tsallis entropy, GP model and ANFIS model performance, which are measured as follows:

$$
\begin{gathered}
M E=\operatorname{Max}\left|P_{i}-O_{i}\right| \\
M A E=\frac{1}{N} \sum_{i=1}^{N}\left|P_{i}-O_{i}\right| \\
R M S E=\sqrt{\frac{\sum_{i=1}^{n}\left(P_{i}-O_{i}\right)^{2}}{n}} \\
N S E=1-\frac{\sum_{i=1}^{n}\left(P_{i}-O_{i}\right)^{2}}{\sum_{i=1}^{n}\left(O_{i}-\bar{O}\right)^{2}}
\end{gathered}
$$

where $O_{i}$ is the observed parameter value, $P_{i}$ predicted parameter value, $\bar{O}$ is the mean value observed parameter value and $n$ number of samples.

\section{Results}

\subsection{Modeling of GP}

In this section, Sensitivity of the GP model for any input parameter is evaluated by adding all four inputs to the models first. Each parameter is then omitted and a total of three separate versions are checked. The GP models used for data on the bed and wall are described as:

For the bed

$$
\begin{gathered}
\operatorname{GPM} \operatorname{Mdel}(1): \frac{b}{B}, \frac{B}{H}, F r, R e \\
G P \operatorname{Model}(2): \frac{b}{B}, \frac{B}{H}, F r
\end{gathered}
$$


For the wall:

$$
\text { GPModel(3): } \frac{b}{B}, \frac{B}{H}
$$

$$
\begin{gathered}
\text { GPModel(1): } \frac{Z}{H}, \frac{B}{H}, F r, R e \\
\text { GPModel(2): } \frac{Z}{H}, \frac{B}{H}, F r \\
\text { GPModel(3): } \frac{Z}{H}, \frac{B}{H}
\end{gathered}
$$

For each channel section, three different models were evaluated to investigate the effect of each input parameter in the GP modeling. The findings of the modeling of bed shear stress show that GP Model (1) had the lowest error consisting of input parameters $(b / B, B / H, F r, R e)$. The results of the modeling of bed shear stress reveal that the lowest error (average RMSE $=0.0874$ ) was observed in the GP model (1) consisting of input parameters $(b / B, B / H, F r, R e)$ and modeled wall shear stress, the GP model (1) has the lowest input error $(z / H, B / H, F r, R e)$ (average RMSE $=0.0692)$, so that the $B / H$ has a major influence on the GP model and validates the effects of model (1). By sensitivity analysis performed, since the flow situation is fully developed, can be ignored the Reynolds number, the parameter has been eliminated in model 2. As shown in Table 3, by omitting Reynolds number $(\mathrm{Re})$ in the input parameters, there is no significant difference. On the other hand, because all the experiments have examined the subcritical flow conditions, can be ignored the effect Froude number, the parameter has been eliminated in model 3. By eliminating the Reynolds number and Froude number parameters, the GP model performance does not change much, and the GP model can be deduced to be insensitive to the $B / H$ parameter. The $B / H$ ratio is obviously important in the estimation of shear stress, as this parameter plays a significant role in the equations stated. Therefore, the model 3 for the bed and wall is chosen as the most suitable model. The results of the most accurate GP model and experimental bed and wall data are shown in the form of the scatter plots in Figures 3 and 4. As seen in statistical analysis, the GP model outcomes are very similar to the bed and wall shear stress line fitted. Dimensionless bed shear stress modeling with GP was superior to dimensionless wall shear stress modeling with average NSE of 0.945 and 0.8266 , respectively, and both models were superior to the other GP models in this study. In order to decide the best answer, the best feedback should be treated as a pattern. Different important parameters in modeling, such as population members, number of generations, tree structures size etc. should be carefully determined in the first step with regard to the consumer of the data examined. The scale of each configuration of the tree will play a major role in the final model's accuracy. Determining the greater numbers than optimal value reduces the precision of the test results prevented from displaying the models are not presented largely because the models generated by genetic programming were very long-scale in order to measure the shear stress. The method of fitting models resulting from genetic programming against experimental results of parameters 2.86, 4.51, 7.14 and

\begin{tabular}{|c|c|c|c|c|c|c|c|c|c|c|}
\hline \multirow{2}{*}{$\mathrm{B} / \mathrm{H}$} & \multirow{2}{*}{ Input variable } & \multicolumn{4}{|c|}{ Bed } & \multirow{2}{*}{ Input variable } & \multicolumn{4}{|c|}{ Wall } \\
\hline & & ME & MAE & RMSE & NSE & & ME & MAE & RMSE & NSE \\
\hline 2.86 & $b / B, B / H$ & 0.2259 & 0.0713 & 0.1051 & 0.9382 & $z / H, B / H$ & 0.0728 & 0.0217 & 0.0870 & 0.7277 \\
\hline 2.86 & $b / B, B / H$, Fr & 0.2445 & 0.1038 & 0.1206 & 0.9456 & $z / H, B / H$, Fr & 0.0693 & 0.0257 & 0.0821 & 0.7759 \\
\hline 2.86 & $b / B, B / H, F r, \operatorname{Re}$ & 0.2338 & 0.0837 & 0.1062 & 0.947 & $z / H, B / H, F r, \operatorname{Re}$ & 0.0363 & 0.0617 & 0.0516 & 0.8021 \\
\hline 4.51 & $b / B, B / H$ & 0.1450 & 0.0962 & 0.0995 & 0.9889 & $z / H, B / H$ & 0.0874 & 0.0530 & 0.0972 & 0.8987 \\
\hline 4.51 & $b / B, B / H$, Fr & 0.1019 & 0.0642 & 0.0638 & 0.9903 & $z / H, B / H, F r$ & 0.0818 & 0.0302 & 0.0890 & 0.8972 \\
\hline 4.51 & $b / B, B / H, \mathrm{Fr}, \operatorname{Re}$ & 0.0927 & 0.0473 & 0.0526 & 0.9911 & $z / H, B / H, F r, \operatorname{Re}$ & 0.0546 & 0.0202 & 0.0701 & 0.8548 \\
\hline 7.14 & $b / B, B / H$ & 0.0826 & 0.0348 & 0.0468 & 0.9955 & $z / H, B / H$ & 0.0589 & 0.1153 & 0.0648 & 0.9049 \\
\hline 7.14 & $b / B, B / H$, Fr & 0.0851 & 0.0408 & 0.0493 & 0.9962 & $z / H, B / H, F r$ & 0.0330 & 0.0321 & 0.0617 & 0.8566 \\
\hline 7.14 & $b / B, B / H, F r, \operatorname{Re}$ & 0.0889 & 0.0466 & 0.0533 & 0.9958 & $z / H, B / H, F r, \operatorname{Re}$ & 0.0422 & 0.0424 & 0.0507 & 0.8982 \\
\hline 13.95 & $b / B, B / H$ & 0.1619 & 0.0908 & 0.1059 & 0.8534 & $z / H, B / H$ & 0.0716 & 0.0926 & 0.1126 & 0.7758 \\
\hline 13.95 & $b / B, B / H, F r$ & 0.2678 & 0.1398 & 0.1566 & 0.8511 & $z / H, B / H, F r$ & 0.0559 & 0.0264 & 0.1117 & 0.8097 \\
\hline
\end{tabular}
13.95 are shown in Figure 4. The statistical analysis results of GP model predictions tabulated in Table 3.

Table 3. Performance metric of GP models to predict SSD 


\begin{tabular}{|c|c|c|c|c|c|c|c|c|c|c|}
\hline 13.95 & $b / B, B / H, F r, \operatorname{Re}$ & 0.2005 & 0.1269 & 0.1376 & 0.8667 & $z / H, B / H, F r, \operatorname{Re}$ & 0.0612 & 0.0720 & 0.1045 & 0.7916 \\
\hline 13.95 & $b / B, B / H$ & 0.2259 & 0.0713 & 0.1051 & 0.9382 & $z / H, B / H$ & 0.0728 & 0.0217 & 0.0870 & 0.7277 \\
\hline
\end{tabular}
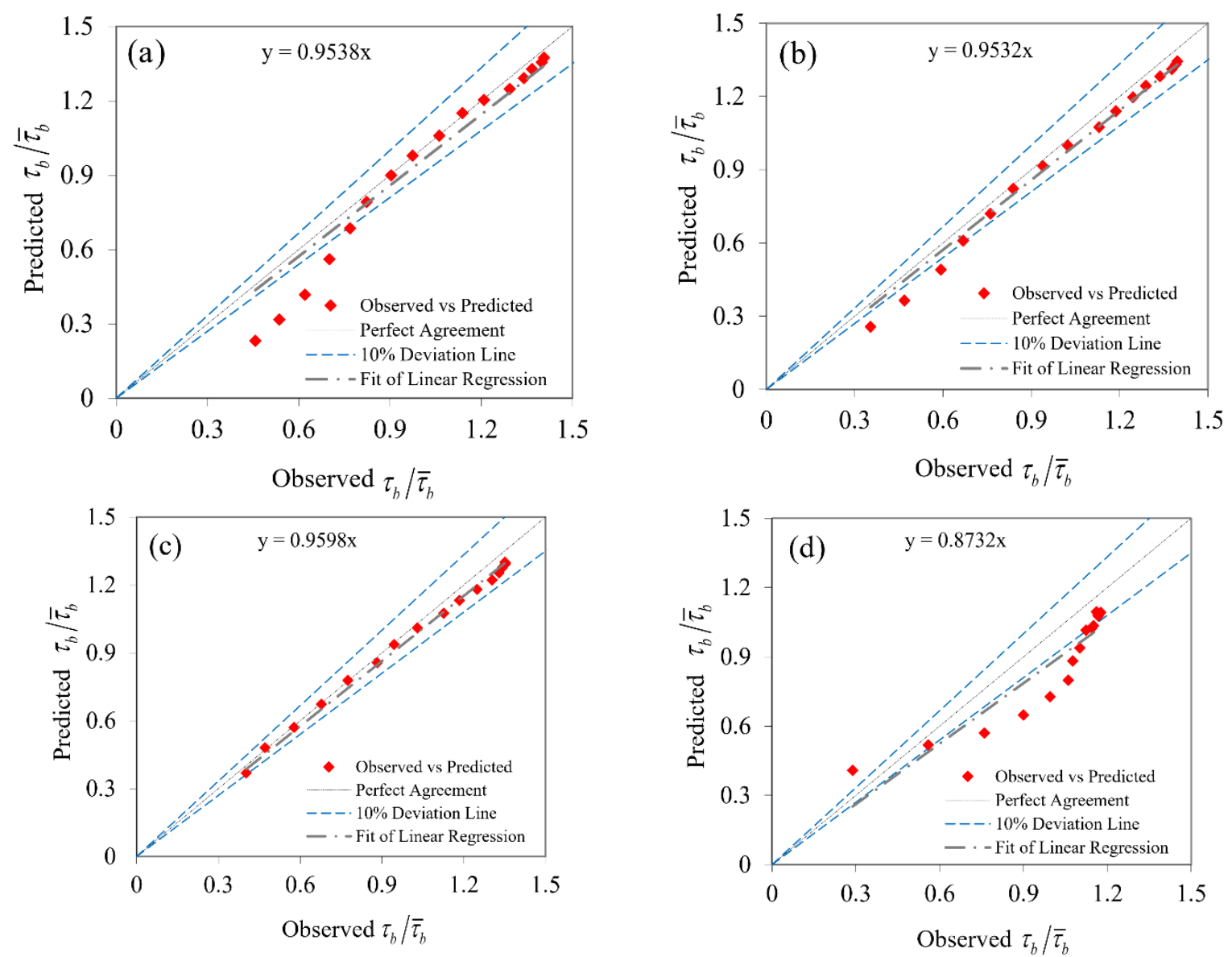

Figure 3. Comparison to the estimate of $\frac{\tau_{b}}{\bar{\tau}}$ between the observed and predicted GP for (a) $B / H=2.86$, (b) $B / H=4.51$, (c) $B / H=7.14$, and (d) $B / H=13.95$.
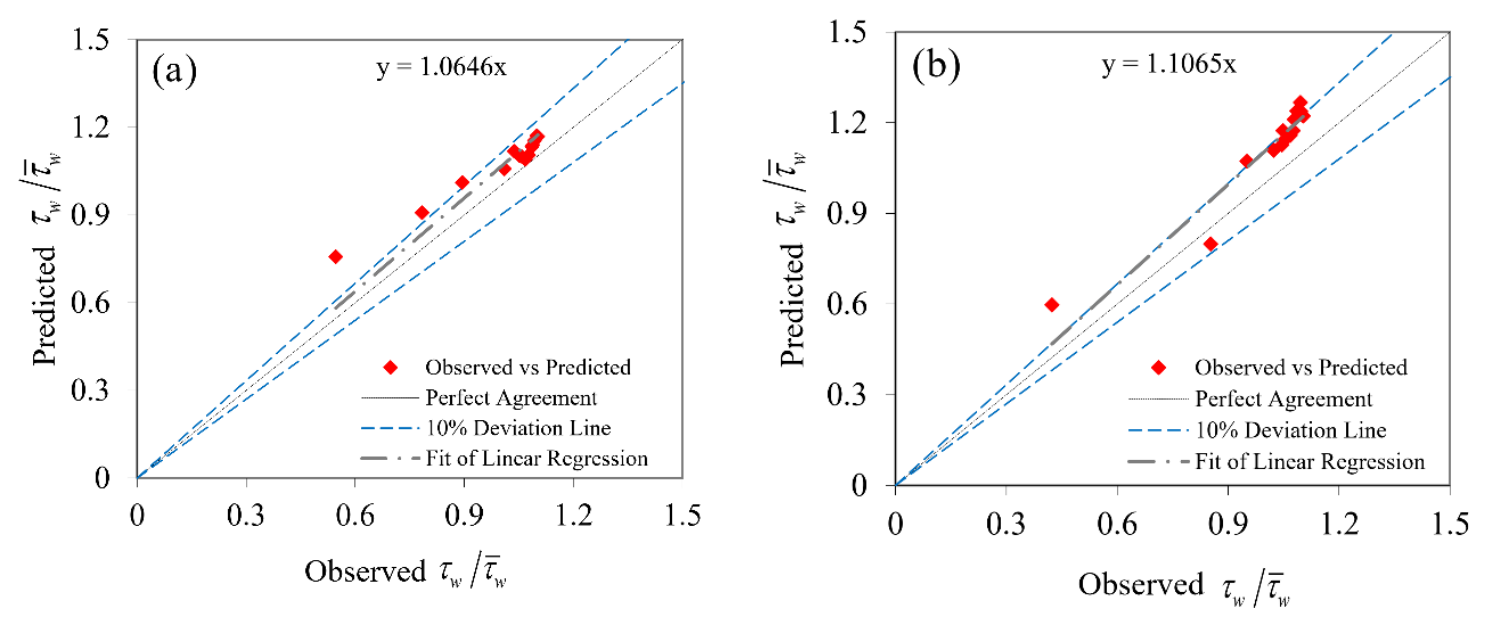

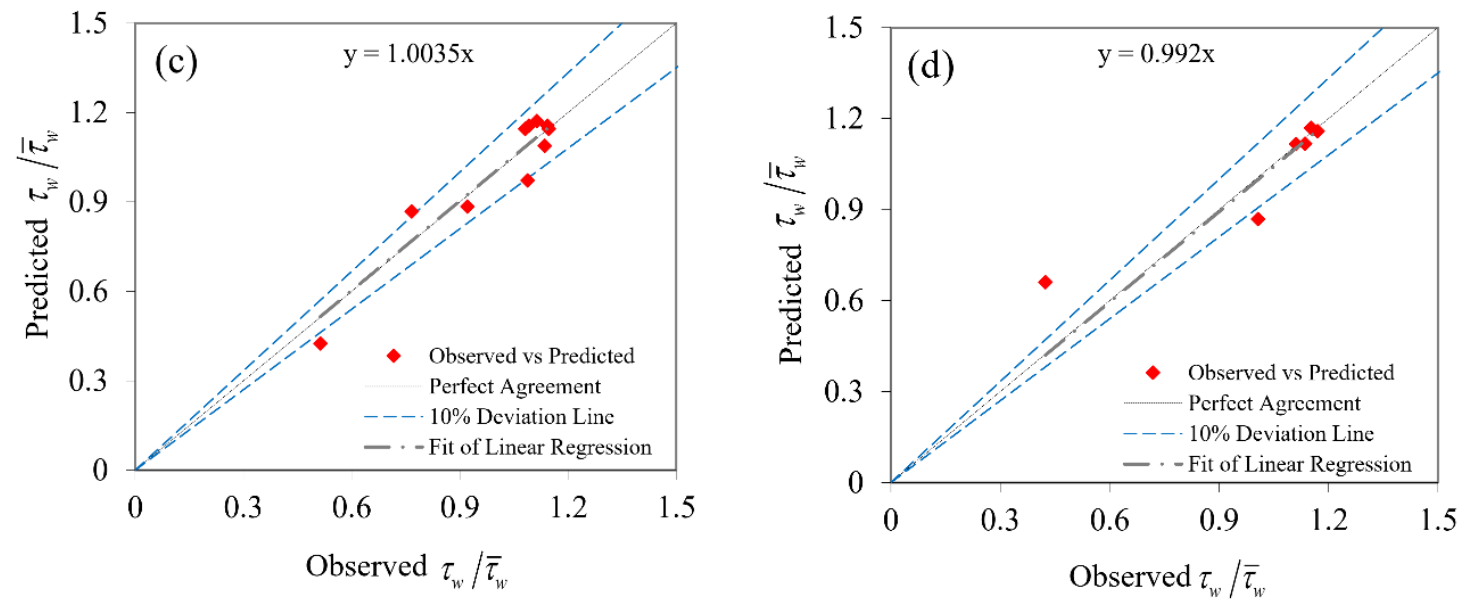

Figure 4. Comparison to the estimate of $\frac{\tau_{w}}{\bar{\tau}}$ between the observed and predicted GP for (a) $B / H=2.86$, (b) $B / H=4.51$, (c) $B / H=7.14$, and (d) $B / H=13.95$

\subsection{ANFIS modeling}

For this purpose, $70 \%$ of the experimental data is used for network training and the remaining $30 \%$ is used for testing results. As input parameters to the model, the parameters $b / B \& B / H$ for bed and $z / H \& B / H$ for wall were presented. Figure 5 shows the performance of the ANFIS model to estimate the bed SSD $\left(\tau_{b}\right)$ and Figure 6 shows the performance of the ANFIS model to estimate the wall SSD $(\tau w), 30 \%$ of the data which were not used in the training stage would be used to evaluate the performance of the model. The results of statistical indexes for modeling shear stress with ANFIS are summarized in Table 4. As well, the estimating bands of the four above parameters using to determine the shear stress are shown in Figure 5. Skewness results obtained from statistical prediction dimensionless parameters.

Table 4. Performance metric of ANFIS model to predict SSD.

\begin{tabular}{|c|c|c|c|c|c|c|c|c|}
\hline \multirow{2}{*}{$\mathrm{B} / \mathrm{H}$} & \multicolumn{3}{|c|}{ Bed } & \multirow[b]{2}{*}{ NSE } & \multicolumn{4}{|c|}{ Wall } \\
\hline & $\mathrm{ME}$ & MAE & RMSE & & $\mathrm{ME}$ & MAE & RMSE & NSE \\
\hline 2.86 & 0.2559 & 0.0991 & 0.1268 & 0.9279 & 0.0383 & 0.0314 & 0.0492 & 0.8026 \\
\hline 4.51 & 0.1728 & 0.1240 & 0.1266 & 0.9744 & 0.0870 & 0.0959 & 0.1004 & 0.9033 \\
\hline 7.14 & 0.2157 & 0.1699 & 0.1724 & 0.9871 & 0.0868 & 0.0634 & 0.0745 & 0.907 \\
\hline 13.95 & 0.2278 & 0.1048 & 0.1271 & 0.8482 & 0.1792 & 0.0909 & 0.1145 & 0.7752 \\
\hline
\end{tabular}
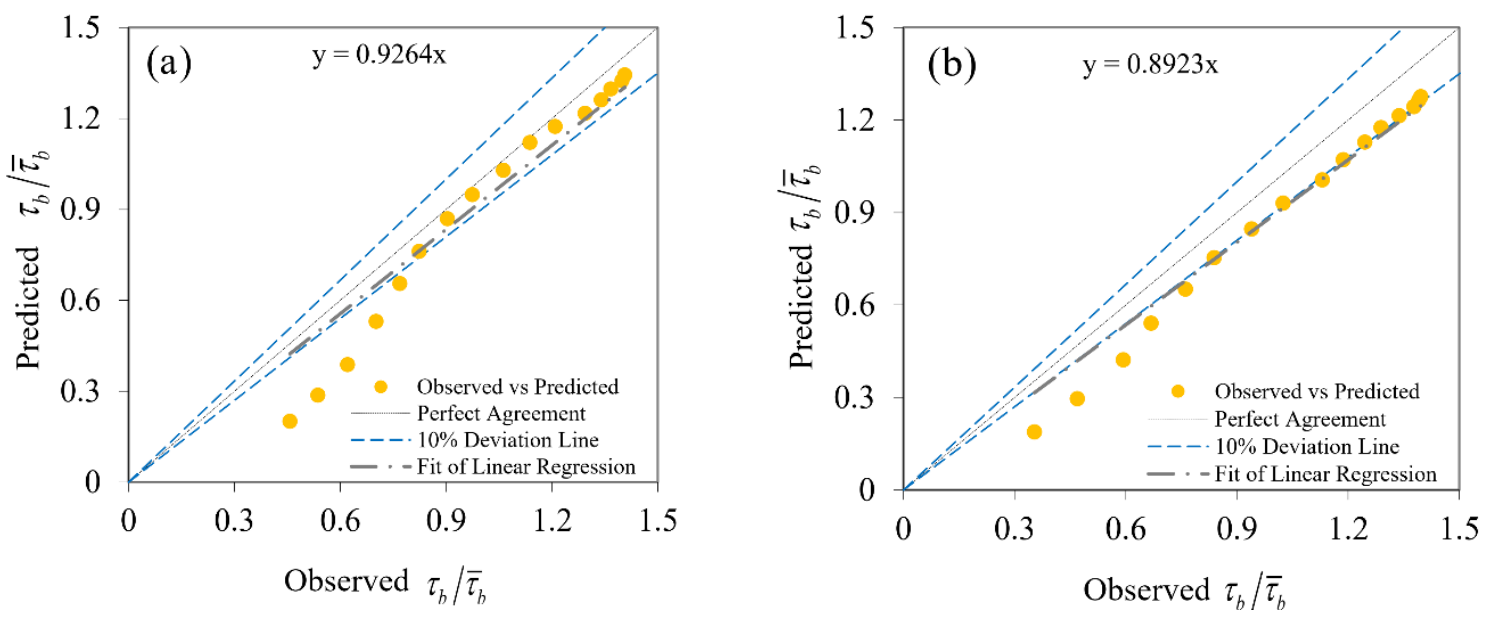

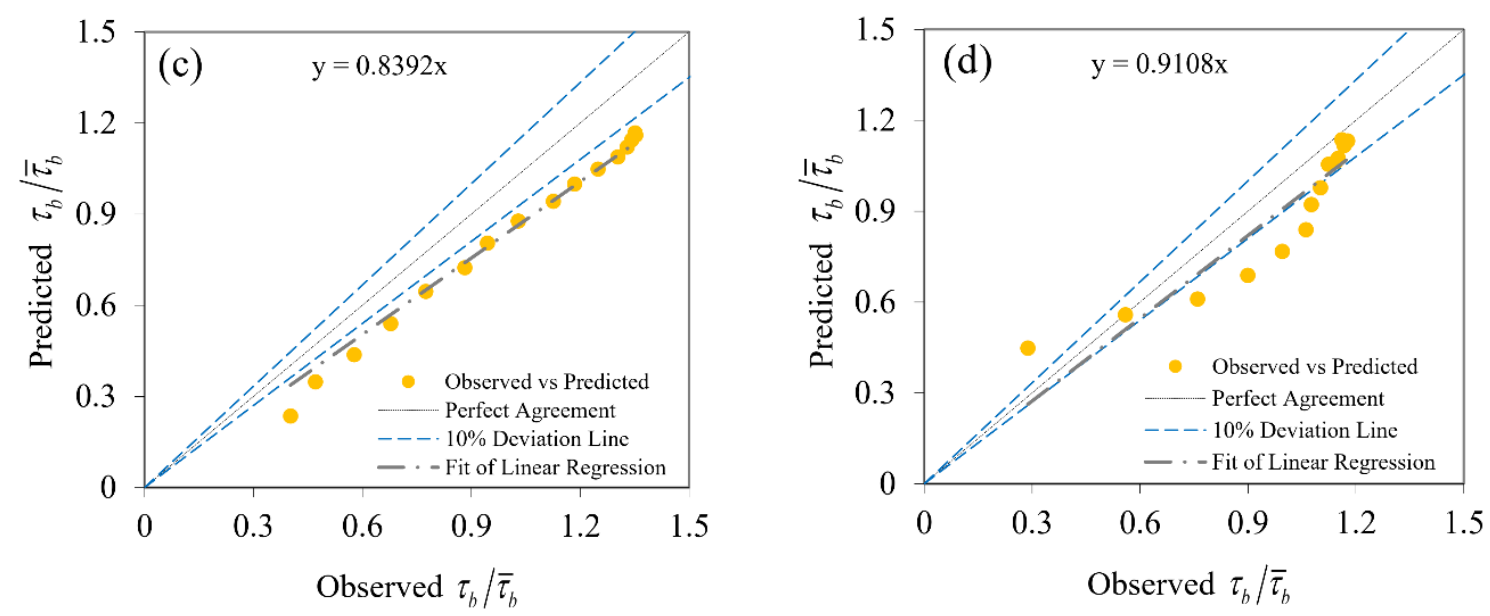

Figure 5. Comparison to the estimate of $\frac{\tau_{b}}{\bar{\tau}}$ between the observed and predicted ANFIS for (a) $B / H=2.86$, (b) $B / H=4.51$, (c) $B / H=7.14$, and (d) $B / H=13.95$.
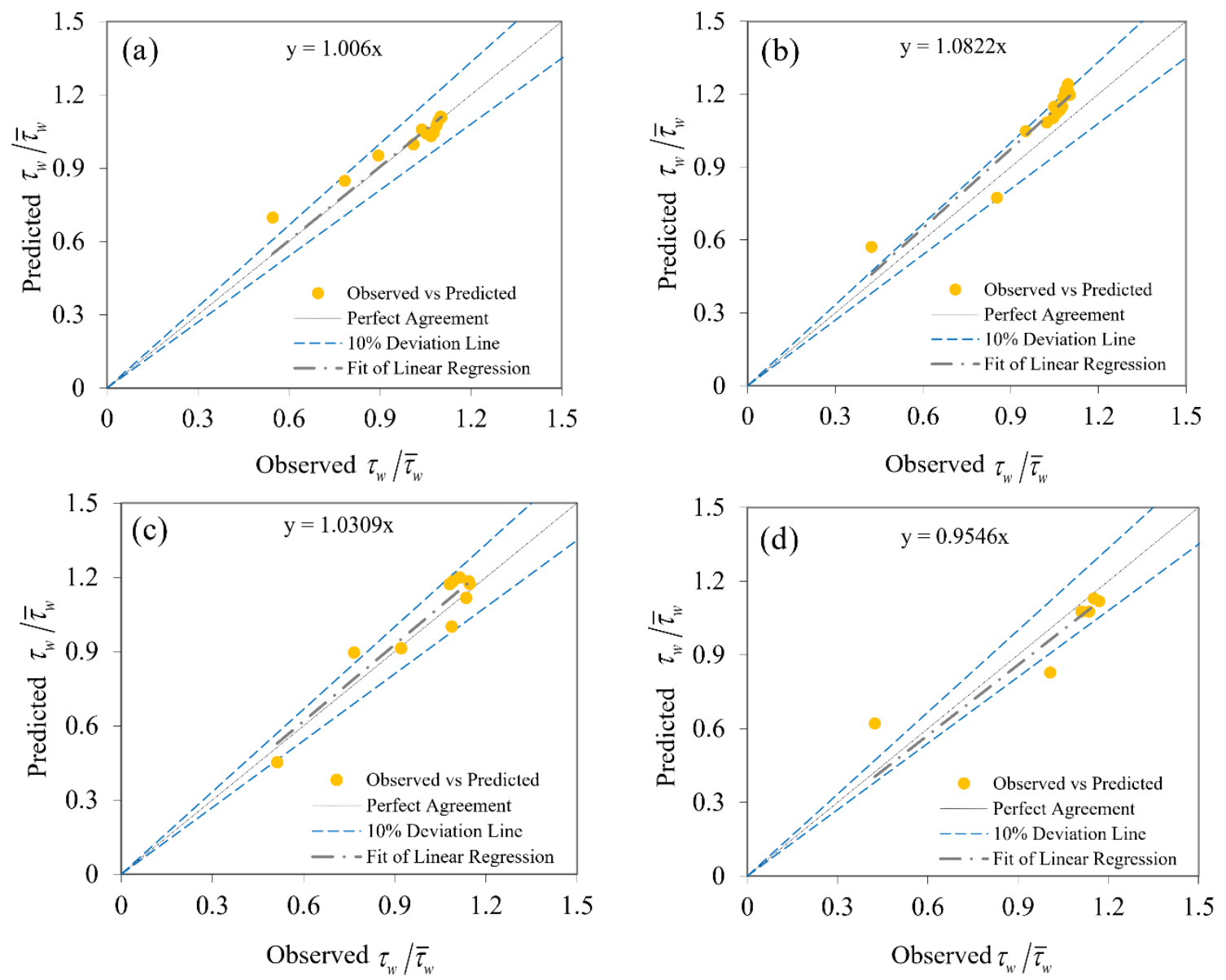

Figure 6. Comparison to the estimate of $\frac{\tau_{w}}{\bar{\tau}}$ between the observed and predicted ANFIS for (a) $B / H=2.86$, (b) $B / H=4.51$, (c) $B / H=7.14$, and (d) $B / H=13.95$.

\subsection{Comparison of the GP model, Tsallis entropy and ANFIS}

The results of the best GP models and Tsallis entropy in shear stress prediction are compared with the experimental results of Lashkar-Ara and Fatahi [8] in this section. Figures 7 and 8 show the experimental results as well as SSD predictions with different models in a smooth rectangular channel for $B / H$ equal to $2.85,4.51,7.14,13.95$. Also, the performance metric of the shear stress estimate by the Tsallis entropy model is shown in Table 5. As 
shown in these statistics, all of the test evidence used to model the SSD using the GP model is realized. For training stage for modeling SSD in rectangular channel using GP model, 70 percent of all data were used, and 30 percent data were used for testing process. As shown in Figure 7 , for $B / H=2.86,4.51,7.14,13.95$, the GP model predicts the bed shear stress better than the Tsallis entropy model. In Figures $8 \mathrm{c}$ and $8 \mathrm{~d}$, for $B / H=7.14 \& 13.95$, the GP model predicts wall shear stress better than the Tsallis entropy model, but in Figures $8 \mathrm{a}$ and $8 \mathrm{~b}$, the Tsallis entropy is more accurately models to predict wall shear stress than the GP model. Additionally, the GP model estimates bed and wall shear stress better than the Tsallis entropy-based model at rising flow depth. It is understandable that the channel architecture is challenging when a model expects higher shear stress values. It is therefore not cost-effective to use the Tsallis entropy method. When the GP model's observations are more accurate, it can be used to design stable channels more consistently. The GP model estimates the bed shear better than the ANFIS model for $B / H=2.86,4.51$, $7.14,13.95$. For $B / H=2.86$, the ANFIS model estimates the shear stress better than the GP model, but the GP model estimates the wall shear stress better than the ANFIS model in $B / H=4.51,7.14,13.95$. The GP model demonstrates superior efficiency to the Tsallis entropy-based model, while both models neglect the influence of secondary flows. It can be inferred that the GP model of bed and wall shear stress estimation is more sensitive than the Tsallis entropy method overestimated the values of bed shear stress and the GP model's outcomes are greater. The bed shear stress values decrease at the middle of the channel (Figure 7), which varies from other situations. From Figures 7 and 8, it can be shown that the GP model's fit line is similar to the 45-degree line than the other ones, and with a higher value of NSE, its predictions are more reliable. In predicting the position of maximal shear stress, both the GP and Tsallis-entropy based models display the same pattern as the centerline of the channel, which is consistent with the experimental outputs.

Table 5. Performance metric of Tsallis entropy to predict SSD.

\begin{tabular}{|c|c|c|c|c|c|c|c|c|}
\hline \multirow{2}{*}{$\mathrm{B} / \mathrm{H}$} & \multicolumn{3}{|c|}{ Bed } & \multirow[b]{2}{*}{ NSE } & \multirow[b]{2}{*}{ ME } & \multicolumn{3}{|c|}{ Wall } \\
\hline & $\mathrm{ME}$ & MAE & RMSE & & & MAE & RMSE & NSE \\
\hline 2.86 & 1.252 & 0.0531 & 0.0706 & 0.9276 & 1.3145 & 0.0622 & 0.0797 & 0.7721 \\
\hline 4.51 & 1.476 & 0.0522 & 0.0625 & 0.9425 & 1.3741 & 0.0749 & 0.0894 & 0.7632 \\
\hline 7.14 & 1.538 & 0.0672 & 0.0685 & 0.9310 & 1.6254 & 0.0631 & 0.0738 & 0.8275 \\
\hline 13.95 & 1.511 & 0.0643 & 0.0840 & 0.8426 & 1.2562 & 0.0893 & 0.1094 & 0.8398 \\
\hline
\end{tabular}
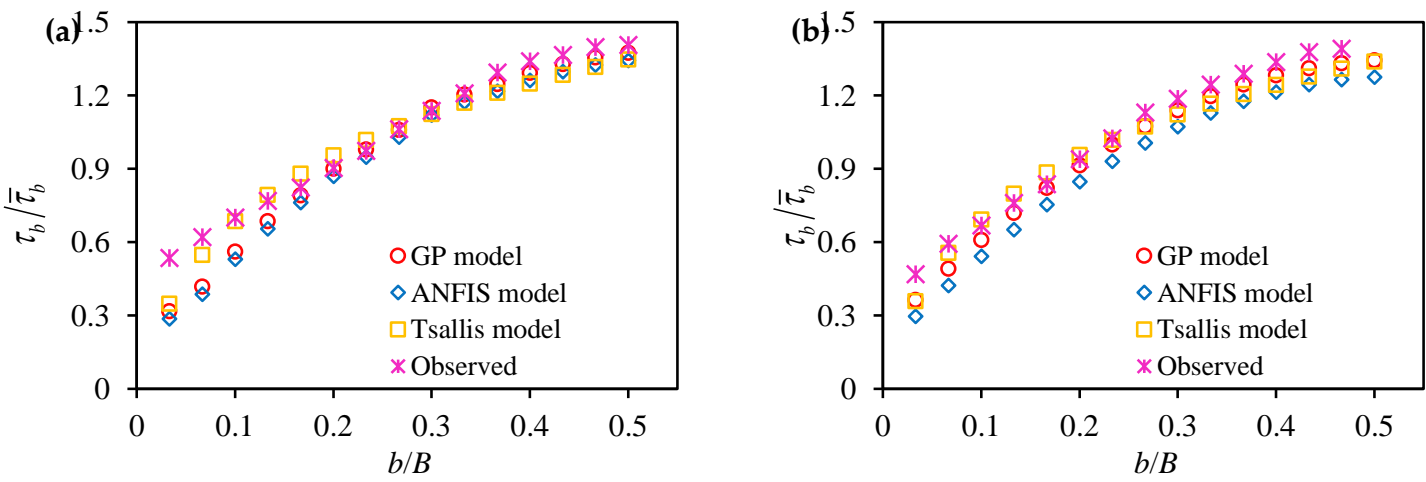

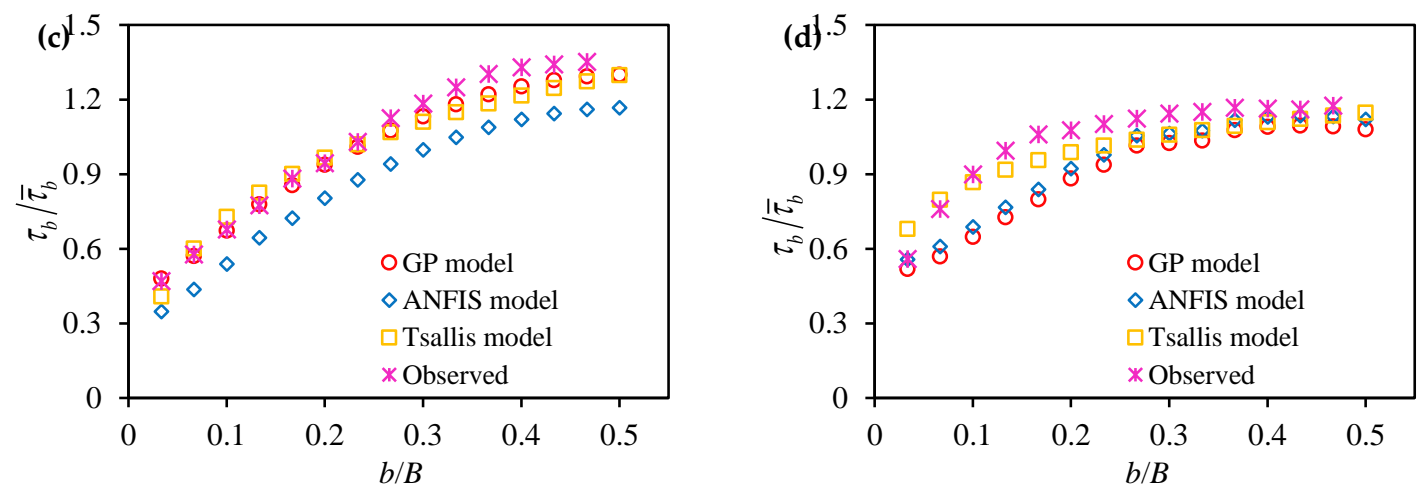

Figure 7. The dimensionless bed shear stress distribution for (a) $B / H=2.86$, (b) $B / H=4.51$, (c) $B / H=7.14$, and (d) $B / H=13.95$.
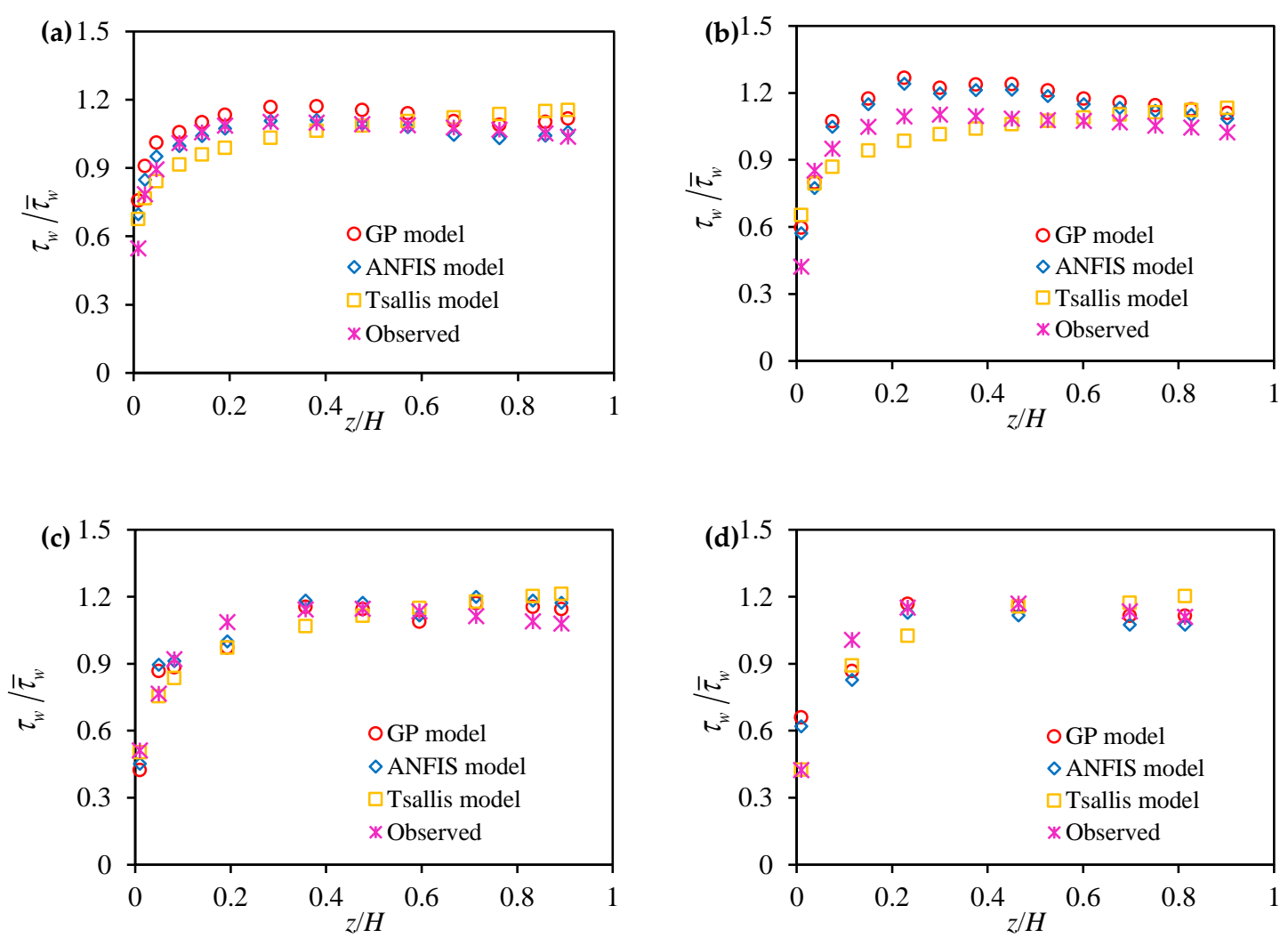

Figure 8. The dimensionless wall shear stress distribution for (a) $B / H=2.86$, (b) $B / H=4.51$, (c) $B / H=7.14$, and (d) $B / H=13.95$.

\section{Conclusion}

The wall and bed shear stresses in a smooth rectangular channel measured experimentally for different aspect ratio. Two soft computing models GP and ANFIS proposed to estimate SSD in rectangular channel. In addition, the results of GP and ANFIS model compared with a Tsallis based equation. Our research has some main findings as follows. 
1. Effect of different input variable on the result is investigated to find the best input combination.

2. In the present study $B / H$ has the highest effect on the prediction power.

3. For bed shear stress predictions, the GP model, with an average RMSE of 0.0893 performed better than the Tsallis entropy-based equation and ANFIS model with RMSE of 0.0714 and 0.138 respectively.

4. To estimate the wall shear stress distribution the proposed ANFIS model, with an average RMSE of 0.0846 outperformed the Tsallis entropy-based equation with an RMSE of 0.0880 followed by the GP model with an RMSE of 0.0904 .

Our finding suggests that the proposed GP algorithm can be used as a reliable and cost-effective algorithm to enhance SSD prediction in rectangular channels.

\section{References}

1. Knight, D.W. Boundary shear in smooth and rough channels. J. Hydraul. Div. 1981, 107, 839-851.

2. Tominaga, A.; Nezu, I.; Ezaki, K.; Nakagawa, H. Three-dimensional turbulent structure in straight open channel flows. J. Hydraul. Res. 1989, 27, 149-173.

3. Seckin, G.; Seckin, N.; Yurtal, R. Boundary shear stress analysis in smooth rectangular channels. Can. J. Civ. Eng. 2006, 33, 336-342, doi:10.1139/105-110.

4. Khodashenas, S.R.; Paquier, A. A geometrical method for computing the distribution of boundary shear stress across irregular straight open channels. J. Hydraul. Res. 1999, 37, 381-388.

5. Pope, N.D.; Widdows, J.; Brinsley, M.D. Estimation of bed shear stress using the turbulent kinetic energy approach-A comparison of annular flume and field data. Cont. Shelf Res. 2006, 26, 959-970, doi:10.1016/j.csr.2006.02.010.

6. Knight, D.W.; Sterling, M. Boundary shear in circular pipes running partially full. J. Hydraul. Eng. 2000, 126, 263275.

7. Park, J.H.; Do Kim, Y.; Park, Y.S.; Jo, J.A.; Kang, K. Direct measurement of bottom shear stress under highvelocity flow conditions. Flow Meas. Instrum. 2016, 50, 121-127, doi:10.1016/j.flowmeasinst.2015.12.008.

8. Lashkar-Ara, B.; Fatahi, M. On the measurement of transverse shear stress in a rectangular open channel using an optimal Preston tube. Sci. Iran. 2020, 27, 57-67, doi:10.24200/sci.2018.20209.

9. Berlamont, J.E.; Trouw, K.; Luyckx, G. Shear Stress Distribution in Partially Filled Pipes. J. Hydraul. Eng. 2003, 129, 697-705, doi:10.1061/(ASCE)0733-9429(2003)129:9(697).

10. Sheikh Khozani; Wan Mohtar Investigation of New Tsallis-Based Equation to Predict Shear Stress Distribution in Circular and Trapezoidal Channels. Entropy 2019, 21, 1046, doi:10.3390/e21111046.

11. De Cacqueray, N.; Hargreaves, D.M.; Morvan, H.P. A computational study of shear stress in smooth rectangular channels. J. Hydraul. Res. 2009, 47, 50-57, doi:10.3826/jhr.2009.3271. 
12. Yang, J.Q.; Kerger, F.; Nepf, H.M. Estimation of the bed shear stress in vegetated and bare channels with smooth beds. Water Resour. Res. 2015, 51, 3647-3663, doi:10.1002/2014WR016042.

13. Martinez-Vazquez, P.; Sharifi, S. Modelling boundary shear stress distribution in open channels using a face recognition technique. J. Hydroinformatics 2017, 19, 157-172, doi:10.2166/hydro.2016.068.

14. Sterling, M.; Knight, D. An attempt at using the entropy approach to predict the transverse distribution of boundary shear stress in open channel flow. Stoch. Environ. Res. risk Assess. 2002, 16, 127-142.

15. Sheikh Khozani, Z.; Bonakdari, H. Formulating the shear stress distribution in circular open channels based on the Renyi entropy. Phys. A Stat. Mech. its Appl. 2018, 490, 114-126, doi:10.1016/j.physa.2017.08.023.

16. Sheikh Khozani, Z;; Bonakdari, H. A comparison of five different models in predicting the shear stress distribution in straight compound channels. Sci. Iran. Trans. A, Civ. Eng. 2016, 23, 2536-2545.

17. Sheikh Khozani, Z.; Hosseinjanzadeh, H.; Wan Mohtar, W.H.M. Shear force estimation in rough boundaries using SVR method. Appl. Water Sci. 2019, doi:10.1007/s13201-019-1056-z.

18. Ardiçlioğlu, M.; Sekçin, G.; Yurtal, R. Shear stress distributions along the cross section in smooth and rough open channel flows. Kuwait J. Sci. Eng. 2006, 33, 155-168.

19. Bonakdari, H.; Tooshmalani, M.; Sheikh, Z. Predicting shear stress distribution in rectangular channels using entropy concept. Int. J. Eng. Trans. A Basics 2015, 28, 360-367, doi:10.5829/idosi.ije.2015.28.03c.04.

20. Rankin, K.L.; Hires, R.I. Laboratory measurement of bottom shear stress on a movable bed. J. Geophys. Res. Ocean. 2000, 105, 17011-17019, doi:10.1029/2000jc900059.

21. Kisi, O.; Dailr, A.H.; Cimen, M.; Shiri, J. Suspended sediment modeling using genetic programming and soft computing techniques. J. Hydrol. 2012, 450-451, 48-58, doi:10.1016/j.jhydrol.2012.05.031.

22. Sheikh Khozani, Z.; Bonakdari, H.; Ebtehaj, I. An analysis of shear stress distribution in circular channels with sediment deposition based on Gene Expression Programming. Int. J. Sediment Res. 2017, 32, 575-584, doi:10.1016/J.IJSRC.2017.04.004.

23. Sheikh Khozani, Z.; Bonakdari, H.; Zaji, A.H. Estimating the shear stress distribution in circular channels based on the randomized neural network technique. Appl. Soft Comput. 2017, 58, 441-448, doi:10.1016/j.asoc.2017.05.024.

24. Tsallis, C. Possible generalization of Boltzmann-Gibbs statistics. J. Stat. Phys. 1988, 52, 479-487, doi:10.1007/BF01016429.

25. Jaynes, E.T. Information theory and statistical mechanics. II. Phys. Rev. 1957, 106, 620.

26. Singh, V.P.; Luo, H. Entropy Theory for Distribution of One-Dimensional Velocity in Open Channels. J. Hydrol. Eng. 2011, 16, 725-735, doi:10.1061/(ASCE)HE.1943-5584.0000363.

27. Jayawardena, A.W.; Muttil, N.; Fernando, T.M.K.G. Rainfall-runoff modelling using genetic programming. In Proceedings of the international congress on modelling and simulation: advances and applications for 
management and decision making; Melbourne, Australia, 2005; pp. 1841-1847.

28. Jang, J.S.R. ANFIS: Adaptive-Network-Based Fuzzy Inference System. IEEE Trans. Syst. Man Cybern. 1993, 23, 665-685, doi:10.1109/21.256541. 